\title{
Review
}

\section{HER2 therapy HER2 (ERBB2): functional diversity from structurally conserved building blocks}

\author{
Ralf Landgraf
}

University of California Los Angeles, Department of Medicine, Hematology-Oncology and Biological Chemistry, Molecular Biology Institute, Los Angeles, California 90095-1678, USA

Corresponding author: Ralf Landgraf, rlandgraf@mednet.ucla.edu

Published: 22 January 2007

This article is online at http://breast-cancer-research.com/content/9/1/202

(C) 2007 BioMed Central Ltd

\begin{abstract}
EGFR-type receptor tyrosine kinases achieve a broad spectrum of cellular responses by utilizing a set of structurally conserved building blocks. Based on available crystal structures and biochemical information, significant new insights have emerged into modes of receptor control, its deregulation in cancer, and the nuances that differentiate the four human receptors. This review gives an overview of current models of the control of receptor activity with a special emphasis on HER2 and HER3.
\end{abstract}

\section{Introduction}

The deregulation of type I receptor tyrosine kinases (RTKs) is a critical aspect of many cancers. In humans this signaling system includes four members: epidermal growth factor receptor (EGFR; ERBB1), human epidermal growth factor receptor (HER)2/neu (ERBB2), HER3 (ERBB3) and HER4 (ERBB4). This review uses the historical HER2 nomenclature in light of the fact that a large body of clinically relevant work has used this identifier, but it should be noted that the Human genome organization Gene Nomenclature Committee approved identifier for HER2 is ERBB2.

Among the various cancers, breast cancers stand out in that not only is over-expression of HER2 a frequent occurrence (20\% to 30\%) in these cancers but also therapeutic targeting of HER2 with humanized antibodies has proven to be a promising approach to treatment. This over-expression of HER2 in breast cancers is accompanied by high levels of constitutively active receptors. Hence, inhibition of this elevated level of constitutive signaling has been a prime objective in the design of therapies. However, notwithstanding the success of therapies targeted against HER2, specifically the use of trastuzumab (Herceptin; Genentech, San Francisco, CA, USA), many mechanistic aspects of
Breast Cancer Research 2007, 9:202 (doi:10.1186/bcr1633)

signaling by HER2 remain poorly understood. The mode of action of Herceptin, which targets the extracellular domains (ECDs) of HER2, is likewise poorly understood and cannot be explained by simple inhibition of ligand-independent receptor association events. (See the paper by Nahta and Esteva [1] for a recent review on the actions of Herceptin.) This limits knowledge-based improvements in therapy and our ability to counteract rapidly emerging resistance (reviewed elsewhere in this series). Part of the problem lies in the fact that type I RTKs, and the ligand binding-deficient HER2 in particular, do not act as autonomous units but as a complex interconnected regulatory system.

Ligand-activated receptor dimers are at the core of signaling by EGFR-type RTKs, resulting in a network of combinatorial receptor interactions. The complexity of this network is further enhanced by a range of ligands with diverse receptor specificity [2]. Once activated, the receptors can signal through at least four alternative pathways, specifically the mitogen-activated protein kinase pathway, phospholipase $\mathrm{C} \gamma$, phosphatidylinositol-3 kinase stimulation of antiapoptotic AKT kinase signaling, and finally STAT (signal transducer and activator of transcription) signaling as the most direct route to transcriptional control [3]. The outcome of signaling can range from cell proliferation to differentiation and apoptosis. These alternate routes to signaling are further modulated by the fact that the complement of signaling partners will not only differ between cell types but also with respect to subcellular localization. However, our understanding of receptor function is largely based on biochemical ensemble measurements. We know next to nothing about the extent to which an individual receptor can engage in signaling in these different pathways simultaneously or sequentially, or whether these signaling events are mutually exclusive at the level of a

$\mathrm{ECD}=$ extracellular domain; EGFR = epidermal growth factor receptor; HER = human epidermal growth factor receptor; IGF = insulin-like growth factor; IR = insulin receptor; NRG = neuregulin; RTK = receptor tyrosine kinase. 

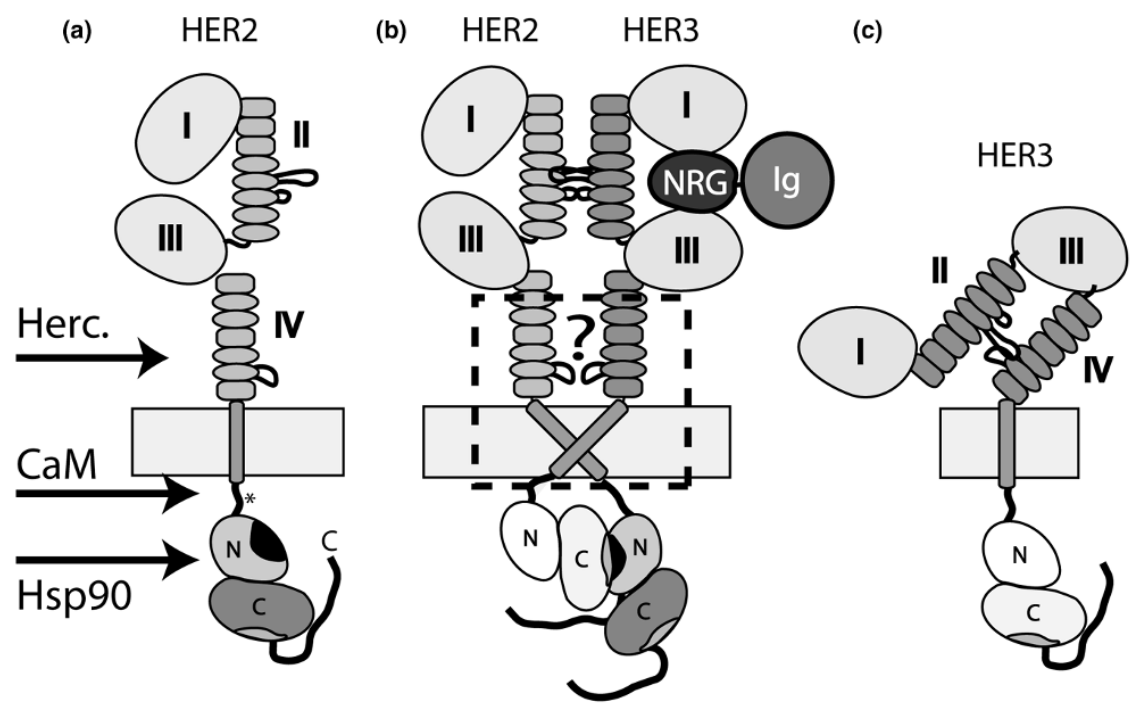

Schematic representation of domains, conformations, and sites of interaction in HER2 and HER3. (a) Domain structure of monomeric HER2, indicating ECDs I to IV with the primary and secondary dimerization loop in the fifth and sixth module of domain II, a single transmembrane span, the cytoplasmic juxtamembrane segment ( ${ }^{*}$ indicates the site of PKC-mediated threonine phosphorylation), the amino- and carboxyl-terminal lobe of the kinase domain, and the carboxyl-terminal tail carrying most adapter binding sites. The sites targeted by Herceptin (Herc.), calmodulin (CaM), and Hsp90 are indicated with arrows. (b) Model of HER2-HER3 heterodimer with bound ligand. NRG indicates the EGF-like domain of neuregulin, bound between domains I and III, and Ig indicates the location of the immunoglobulin-like amino-terminal domain of neuregulins. The receptor dimer is stabilized by reciprocal interactions between domains II of both receptors. The physical separation of domains IV in the diagram does not necessarily indicate physical distance but is meant to emphasize that based on experimental data, and in contrast to transmembrane span packing, domain IV interactions do not contribute significantly to dimer stabilization. The exact nature of interactions by both components (boxed with dashed lines) is not clear at this point. The indicated interactions of the cytoplasmic kinase domains summarize the recently proposed mode of allosteric activation based on EGFR structures [38]. (c) HER3 in the closed/locked conformation, stabilized by an intramolecular tether involving the primary dimerization loop in domain II and its structural equivalent in domain IV. ECD, extracellular domain; EGFR, epidermal growth factor receptor; HER, human epidermal growth factor receptor; PKC, protein kinase C.

single receptor. This limitation in our understanding is of course not unique to HER2 signaling but should be kept in mind, especially as we attempt to interpret results indicating signaling complexes that involve large protein scaffolds.

Much of our mechanistic knowledge on RTK signaling is based on EGFR, which is by far the best studied member in this family. However, EGFR differs in many key aspects from HER2. This review emphasizes mechanistic foundations of type I RTK signaling, derived from structure and function analyses of all four family members, with an emphasis on HER2.

\section{The basic paradigm of ligand-induced dimerization and transphosphorylation}

All four EGFR-type RTKs share a very conserved structural framework (Figure 1a), which consists of four ECDs, a single transmembrane span, a cytoplasmic juxtamembrane linker region, a tyrosine kinase component, and a carboxyl-terminal tail. This carboxyl-terminal tail is the main substrate of activationdependent tyrosine phosphorylation and subsequent recruitment of adapter proteins, although tyrosine phosphorylation has also been reported in the kinase domains itself $[4,5]$. The basic paradigm for activation control centers on ligand- induced homo- and hetero-dimerization of receptors, followed by tyrosine phosphorylation of the cytoplasmic portions of the receptors in trans. Based on confirmed receptor tyrosine phosphorylation sites, a recent microarray-based study determined the ability of such tyrosine phosphorylatedpeptides to recruit $\mathrm{SH} 2$ or phosphotyrosine-binding domains [4]. This study revealed significant differences between the four RTK family members. Although HER3 exhibited few changes in its recruitment pattern as a function of peptide ('bait') concentration, significant changes occurred for HER2 suggesting that the complement of adapter proteins recruited by activated HER2 would qualitatively vary significantly more as a function of the levels of activated receptors. In addition, a projection for different receptor pairs showed that the HER2/HER3 heterodimer outperforms other RTK combinations in terms of the range of recruited adapters and its ability to carry out efficient recruitment at low to medium concentrations of tyrosine phosphorylated sites. This is consistent with earlier observations that the HER2/HER3 heterodimer represents the most potent mitogenic signaling pair [2].

The unique nature of the HER2/HER3 receptor pair in signaling is also reflected in the basic mode of activation, 
which features a 'separation of duties', as compared with the basic model established by homodimers of EGFR. Although EGFR and HER4 can form fully functional homodimers, HER3 has impaired kinase activity [6] and relies on the kinase activity of its heterodimerization partners for activation [7]. HER2 fails to bind any of the known RTK ligands but contributes its kinase activity to all possible heterodimers. Although HER2 itself does not bind any known ligand, it is believed that many of the molecular details in receptor interactions, established for homodimers of ligand-bound EGFR, extend to heterodimers of HER2 with its ligand binding partners, primarily EGFR and HER3.

The different RTKs exhibit preferential binding to subsets of ligands, with EGFR binding EGF and related ligands whereas HER3 and HER4 bind neuregulins (NRGs; also referred to as heregulins). NRGs are a large family of ligands arising from alternative splice variants of four NRG genes [8]. NRGs and EGF-type ligands share an essential and structurally almost identical EGF-like domain, and are generated by proteolytic processing of membrane bound precursors. Enhanced signaling via the HER3/HER2 receptor pair is one of the routes of resistance encountered in EGFR-targeted therapies. The inhibition of the ADAM protease-mediated proteolytic processing of the cell surface precursors of NRGs has recently been evaluated as a drug target in non-small-cell lung cancers that showed resistance to the EGFR kinase inhibitor gefitinib (Iressa; AstraZeneca, Wilmington, DE, USA) [9]. This study identified NRG processing as a promising point of therapeutic intervention. In contrast to EGF, where processing occurs amino- and carboxyl-terminal of the peptide-size EGF domain, all known mature NRGs retain additional but diverse domains amino-terminal of the EGF-like domain that are not required for activation on the basic level of receptor dimers. However, these additional amino-terminal domains, or the presence of unrelated domains of comparable size, are required for efficient receptor downregulation and signal attenuation [10]. Because this enhancement in signal attenuation correlates with an enhanced disruption of previously observed higher order oligomers of HER3 receptors [11], this may provide a glimpse into the additional control mechanisms that act upon RTK receptors on the level of higher order association states, which arguably is one of the least understood aspects of RTK signaling at this point.

Insight into the mode of ligand binding and receptor interaction comes from crystal structures of the ECDs with and without ligand. Several in-depth reviews of these studies are available [12,13]. Structurally, all four RTK receptors contain four ECDs. Domain I (also named L1) is structurally highly homologous to domain III (L2; each approximately 170 residues), and domain II (C1 or $\mathrm{S} 1$ ) is homologous to domain IV (C2 or S2; each approximately 140 residues). Ligand binding occurs on the 'outside' of dimers, not the receptordimer interface, and involves interactions with domains I and
III (Figure 1b), both of which have a $\beta$ helical structure. However, the relative contribution by domains I and III to ligand binding differs between receptors, with domain III being the main contributor for EGF binding to EGFR [14] whereas domain I is the dominant site of interaction for NRG binding to HER3 $[15,16]$ and HER4 [17].

In the structure of ligand-free HER2 ECDs, domains I and III interact with each other in a fashion that would block ligand access, in part explaining the lack of ligand binding by HER2. Whether this feature is truly unique to HER2 will remain unknown until the structures of other family members in their ligand-free and extended conformations are resolved. Currently available, ligand-free structures for EGFR and HER3 demonstrate the receptors to be in a significantly different and tethered conformation, which is likely to be in equilibrium with an extended conformation similar to that observed for HER2. The cysteine-rich domains II and IV also feature a high degree of structural similarity, especially with respect to their individual laminin-like modules, which are arranged in a linear fashion. However, this similarity is deceiving. When they are individually classified based on the nature of their disulfide bonding, domains II and IV differ in the number and subtype of laminin-like modules [12] (shown as differences in module shape in Figure 1a). More importantly, the contribution of both domains to receptor function appears to be very distinct.

The crystal structures of the ECDs of EGFR with bound ligand outline key aspects of ligand binding and receptor dimerization [18,19]. However, in both crystal structures domain IV was either not resolved or had to be removed to facilitate crystallization. Domain II directly stabilizes the dimer through interlocking dimerization loops of two interacting receptors. Those primary dimerization loops protrude from the fifth module of domain II. This interaction was not detected in the absence of ligand for soluble constructs consisting of only domains I to III [20], suggesting that interactions through this interface are very weak in the absence of ligand-induced conformational changes because the ligand itself is not part of the dimer interface. Additional interactions occur through a smaller loop protruding from the sixth module of domain II, and those interactions have been implicated in providing discrimination for the formation of the correct heterodimers and the enforcement of ligand-induced conformational changes in the activation process [21]. Although domain IV is structurally similar to domain II, mutagenesis of domain IV indicates that it does not contribute significantly to stabilizing dimers [21]. This observation is consistent with the inability of domain IV to assume a stable and defined conformation in crystals of EGFR-ECD dimers.

The ECDs can therefore be divided into two functional units: domains I to III, containing ligand binding and key dimerization controls, and a functionally distinct domain IV. This 
classification resembles the evolutionary related insulin receptor (IR) as well as the insulin-like growth factor (IGF)1 receptor, despite large differences in the nature of ligands for both RTK families. Indeed, the degree of structural conservation between the first three domains of $I R$ and EGFR-type RTKs is very high [22]. However, domain IV is absent in IR/IGF1R and is functionally replaced with a second polypeptide chain ( $\beta$ ). The $\beta$-chain of IR/IGF1R establishes covalent links to the $\alpha$-chain (domains I to III) and between $\beta$-chains of two receptors, thereby generating a covalently linked dimer. Given that domain IV is the most significant point of departure between the otherwise very homologous IR and EGFR family of RTKs, it will be interesting to see in the future what this segment contributes to function because it is likely to serve a purpose in controlled signaling beyond that of a mere spacer. Because it is domain IV of HER2 that is the target of Herceptin binding [23], this lack of understanding of the exact contribution by domain IV also complicates current efforts to place the activity of Herceptin on a mechanistic foundation.

Although the mechanistic contribution of domain IV in the ligand-bound receptor dimer is currently not clear, domain IV is a critical component in the formation of an intramolecular tether between the 'dimerization loop' in domain II and a similar loop in domain IV (Figure 1c). This intramolecular 'tether' or 'lock' was first observed in the crystal structure of the ECDs of HER3 [24] and subsequently in several structures of EGFR, and HER4 [25-27]. In contrast, the ECDs of HER2 represent the only example thus far in which all four ECDs crystallized as a monomer in an extended conformation, representing a structure expected to be similar to that of individual receptors in a receptor dimer. The dimerization interface in the tethered conformation is not available for intermolecular interactions, and the ligand binding pocket between domain I and III is disrupted. This provided a very attractive and structure-based model for receptor activation control in which the intramolecular tether presents a barrier to spontaneous dimerization and activation [12]. The inability of HER2 to adopt this 'autoinhibited' conformation was expected to result in a receptor, predisposed to uncontrolled dimerization and signaling, which correlates nicely with the high transforming potential of HER2 [28]. However, although the inability of HER2 to assume an autoinhibited conformation may contribute to its potency, subsequent mutagenesis of the tether in EGFR $[19,29]$ and HER3 ECDs (evaluated in the context of an HER3-HER2 chimera) [11] surprisingly showed little impact of a defective tether on the suppression of basal activation. For soluble ECDs of EGFR, the release of the tethering mechanism results in a modest increase in the affinity for EGF [25], consistent with an increased availability of ligand binding sites in the extended conformation. Recent modeling and direct measurements of the impact of the tether on ligand binding in a cellular setting likewise indicated only a modest twofold increase in ligand binding affinity [30].
However, the presence of the tether in several crystal structures of EGFR, HER3, and HER4 strongly suggests that this conformation is biologically relevant. Interestingly, two crystal structures (ECDs of EGFR and HER4) were obtained at $\mathrm{pH} 5.0$ to $5.5[25,27]$, a $\mathrm{pH}$ range comparable to the environment of late endosomes. This highlights another aspect of ligand binding: $\mathrm{pH}$ dependency. At endosome $\mathrm{pH}$, EGFR loses most of its affinity for EGF or transforming growth factor- $\alpha$ (but not betacellulin), whereas HER3 and HER4 retain significant ligand binding affinity $[16,27]$. Surprisingly, the ability of HER3 to bind ligand at low pH is even further enhanced in the locked/tethered conformation [16]. This is of interest not only for HER3 but also for HER2, because the endosome is emerging as a compartment not only for downregulation and sorting but also for signaling. In the case of EGFR, signaling can be initiated from endosomes [31], and the endosomal protein P14 is needed to recruit the mitogen-activated protein kinase scaffolding protein MP1 [32]. Although HER2 itself is apparently deficient in stable tether formation, conditions that favor the adaptation of the tethered conformation by its heterodimerization partners are expected to shift the equilibrium between HER2 containing heterodimers and dissociated receptor complexes in favor of dissociated receptors. This may alter the recruitment of adapter proteins and influence receptor routing. Hence, the tethered conformation, whose role on the cell surface is not yet clear, may potentially play a more prominent role in modulating receptor actions in endosomal compartments.

\section{Beyond dimerization: additional control mechanisms within receptor complexes}

Whether receptor dimerization is sufficient for activation was questioned in the past [33], and several studies challenged the model of purely association driven activation. Although dimerization of engineered intermolecular disulfides can activate EGFR [34], similar experiments using HER2 have not resulted in activated receptors [35], indicating that spatial proximity alone is not sufficient to ensure efficient phosphorylation. Likewise, cytoplasmic kinase segments fused to strongly dimerizing transmembrane spans alone do not result in activation. Cysteines placed at various positions throughout the membrane spanning helix of EGFR identify preformed dimers with distinct interfaces in the active and inactive state, demonstrating preformed but active receptor dimers and suggesting rotational transitions in the packing of the transmembrane span upon activation [36]. In contrast, the loss of the ECDs of HER2 in the context of its natural transmembrane domains has long been known to result in constitutive activation [37]. Recent experiments in which kinase domains were artificially anchored to a membrane surface likewise resulted in active kinases [38]. The overall picture that appears to emerge from a broad range of studies suggests that spatial proximity or dimerization is sufficient for activation when intrinsic control mechanisms have been disabled through mutation, truncation, or out-of-context fusion of kinase domains, whereas dimerization alone is not sufficient when intrinsic control mechanisms are retained. 
While dimerization of intact receptors alone appears to be insufficient for activation, it may also not be the end-point in receptor association states. The role of higher order complexes beyond receptor dimers is of special relevance for HER2. Activation of HER2 in the context of heterodimers of HER2 and HER3 results in tyrosine phosphorylation of both receptors. Given the kinase deficient nature of HER3, this observed activation of HER2 has been ascribed to trans phosphorylation in the context of higher order association states for the ligand-bound heterodimers [39]. However, the recent model for activation, discussed below, allows for a simpler model of intramolecular phosphorylation of activated HER2 as far as only the dominant tyrosine phosphorylation of the carboxyl-terminal tail is concerned [40]. The kinasedeficient HER3, on the other hand, exhibits elevated selfassociation behavior in the absence of ligand, but not only does it fail to form stable ligand-induced dimers [41] but also its ligand-independent self-association is destabilized by ligand binding [11]. This suggests that a complex equilibrium of pre-associated inactive receptor complexes, active dimers, and monomers is likely to exist on the surface of cells.

The transmembrane span of HER2 has long been implicated as a key contributing factor to self-association and activation control. The self-association capacity of the transmembrane spans correlates with the degree of conservation of GXXXG motifs, present in two copies and various levels of conservation in all four receptors [42]. Surprisingly, the well known activating mutation of the transmembrane span (V659E, Neu), which alters one of the GXXXG motifs, destabilizes interactions and has been suggested to shift the equilibrium of two alternative packing modes representing the active and inactive state of the receptor [43]. The 'Neu' mutation may therefore not activate by enhancing dimerization but by increasing the probability at which transiently stable complexes, resulting from random collisions, switch stochastically into an active state. Although this model requires further confirmation, critical contributions of the transmembrane span in signaling are also confirmed by the fact that chimera of the ECDs of HER3 and the cytoplasmic domains of HER2 are only functional when connected through the stronger self-associating transmembrane span of HER2 [11] but not with the corresponding segment of HER3 [41]. This is consistent with the observation that the transmembrane span of HER3 exhibits the weakest selfassociation [42]. This further indication for a need for conformational changes within the receptor complexes also underscores the importance of comparisons with the evolutionary related IR/IGF1R system in which the covalent nature of the receptor dimers clearly necessitates additional allosteric control mechanisms.

Recent crystallographic studies have provided fascinating insights as to what allosteric activation might mean at the level of the final receiving module, namely the kinase domain. With the exception of crystals obtained in the presence of the type II (inactive state) kinase inhibitor lapatinib, the kinase domain of EGFR assumes an active conformation in all other available crystal structures. This is consistent with the observation that EGFR, in contrast to IR and indeed most protein kinases, does not require phosphorylation of its activation loop for activation.

Instead, recent studies conducted by Kuriyan and colleagues [38] show that EGFR kinase domains achieve allosteric activation by assuming a dimeric packing mode that resembles interactions observed in the CDK2/cyclin $A$ complex [40]. In this model, two kinase domains form an activated complex through the interaction of the carboxyl-lobe of one kinase domain with the amino-lobe of a second kinase domain. This destabilizes an inhibitory wedge between leucines in the activation loop and the adjacent $\alpha \mathrm{C}$ helix in the amino-lobe of the kinase domain. This inhibitory control is also lost in the heterozygous mutations L834R and L858R, found frequently in EGFR in lung cancers. The identified interface between kinase domains also includes the carboxylterminal 'LVI' motif in EGFR, HER3 and HER4 ('VVI' in HER2), which was known to be required for the formation of catalytically competent heterodimers of HER2 and HER3 [44]. Although HER3 lacks conservation of the interface on the amino-terminal lobe, which is needed to receive an activating signal, it fully conserves its carboxyl-terminal interface, presumably enabling it to act as an allosteric activator for HER2 or EGFR (Figure 1b). This provides an explanation for the overall conservation of the catalytically dead kinase domain of ERBB3. Interestingly, deletions of portions of the carboxyl-terminal tail segment result in a release of intrinsic inhibitory blocks. The carboxyl-terminal tail is an intrinsic part of an alternative, symmetric dimer interface between kinase domains [45]. This interaction may therefore present an alternative inhibitory packing mode.

The available model for allosteric cross-activation provides a clear mechanistic foundation. However, in order to better understand how this model can be reconciled with the observation that preformed dimers can exist in either active or inactive states, we will need further structural information on the components that link the ligand binding domains to the kinase domains and on the interplay of those modules throughout the activation process. How allosteric changes may be passed on from the ECDs to the interior, once dimers are formed, is not clear at this point. However, the model of alternative transmembrane packing modes provides one attractive option [43].

All of the models presented above attempt to explain the basic signaling behavior of HER2 either based on an individual receptor or complexes between RTKs. However, although activation results in the recruitment of various adapter proteins to the receptors, it is important to realize that HER2 is not free of cytoplasmic associations in its nonactivated state. Specifically, HER2 is stabilized by the 
chaperone Hsp90. Beyond stabilizing mature, cell surface HER2, Hsp90 also interferes with the heterodimerization of HER2 and HER3 [46], and the conserved recognition motif for Hsp90 [47] overlaps with the recently discovered allosteric activation interface in the amino-terminal lobe of the HER2 kinase domain. This poses interesting and as yet unresolved questions with regard to the order of events in HER2 activation.

In addition, the juxtamembrane region of EGFR associates with calmodulin in a manner that is regulated through threonine phosphorylation (Thr654) by protein kinase C [48]. Such an association with calmodulin has also been demonstrated for HER2 [49]. This would dramatically change the charge composition of the juxtamembrane section, which has been proposed to contribute to activation control [50]. Calmodulin binding would also alter the flexibility of the juxtamembrane linker region. Such an addition of rigidity may be of relevance in the context of the proposed allosteric activation scheme for RTK kinase domains and the mode of transmission of conformational changes from the exterior to the interior.

However, how these additional control mechanisms act in a concerted manner under normal ligand-controlled conditions, and how their alterations may factor into the loss of control in over-expressing cancers is not clear at this point.

\section{Conclusion}

Structural information on the mode of interaction between EGFR-type RTKs has provided a wealth of information on a mechanistic level and satisfactory explanations for the mode of action of some of the second-generation monoclonal antibodies. Binding of cetuximab (Erbitux, ImClone Systems, New York, NY, USA) to domain III of EGFR interferes with ligand binding and the ability of the receptor to adopt an extended conformation [26], and pertuzumab (2C4, Omnitarg, Genentech, San Francisco, CA, USA) targets the dimerization motif in domain II of ERBB2 [51], thereby preventing the formation of signaling competent heterodimers. Additional antibody-based therapeutics are currently under investigation but the direct correlation between now established mechanistic features of RTK signaling and antibody activity is not always as clear. This remains to be the case for Herceptin (trastuzumab), although one of its properties - the interference with the generation of the hyperactive cleavage product of HER2 (p95HER2) [52] can be reconciled nicely with the available co-crystal structure of HER2 and Herceptin.

Combined structural and biochemical studies have also shown that the established model of ligand-induced dimerization correctly identifies an essential stage in receptor control but is insufficient to provide a complete picture of the control mechanism within this complex signal transmission device represented by EGFR-type receptors. On the one
This article is part of a review series on HER2 therapy, edited by Mark Pegram.

Other articles in the series can be found online at http://breast-cancer-research.com/articles/ review-series.asp?series=BCR_HER2

hand, we currently lack sufficient mechanistic insights into the coupling of external ligand binding events to kinase domain activation, and receptor dimerization alone is clearly not sufficient. On the other hand, we have in the intramolecular tether a structurally very well defined control mechanism in search of its proper place in the overall scheme of receptor control. This complexity of receptor intrinsic control mechanisms is superimposed on secondary layers of control such as receptor associating factors such as Hsp90 or calmodulin, and subcellular localization such as membrane microdomain partitioning. A better understanding of these different levels of control will certainly not only lead to better targeted and mechanistically based drugs, but should also provide insight into the mechanisms underlying emerging resistance as well as mechanisms that lead to differences in the ultimate outcome of signaling. The latter may ultimately allow us not only to think of RTKs in general, and HER2 in particular, as overactive receptors to be inhibited, but also may teach us how to actively redirect the signaling of overexpressed HER2 receptors into a proapoptotic direction, given that apoptosis is part of the repertoire of signaling outcomes for HER2.

\section{Competing interests}

The author declares that they have no competing interests.

\section{Acknowledgments}

Dr Feng Guo provided valuable feedback in the creation of this manuscript. Support was provided by the $\mathrm{NIH} / \mathrm{NCl}$ (CA098881) and the Susan B Komen Breast Cancer Foundation (BCTR0504291).

\section{References}

1. Nahta R, Esteva FJ: Herceptin: mechanisms of action and resistance. Cancer Lett 2006, 232:1 23-138.

2. Pinkas-Kramarski R, Soussan L, Waterman H, Levkowitz G, Alroy I, Klapper L, Lavi S, Seger R, Ratzkin BJ, Sela M, et al.: Diversification of $\mathrm{Neu}$ differentiation factor and epidermal growth factor signaling by combinatorial receptor interactions. $E M B O$ J 1996, 15:2452-2467.

3. Rubin I, Yarden Y: The basic biology of HER2. Ann Oncol 2001, Suppl 1:S3-S8.

4. Jones RB, Gordus A, Krall JA, MacBeath G: A quantitative protein interaction network for the ErbB receptors using protein microarrays. Nature 2006, 439:168-174.

5. Bose R, Molina H, Patterson AS, Bitok JK, Periaswamy B, Bader JS, Pandey A, Cole PA: Phosphoproteomic analysis of Her2/ neu signaling and inhibition. Proc Natl Acad Sci USA 2006, 103:9773-9778.

6. Guy PM, Platko JV, Cantley LC, Cerione RA, Carraway KLR: Insect cell-expressed p180erbB3 possesses an impaired tyrosine kinase activity. Proc Natl Acad Sci USA 1994, 91: 8132-8136.

7. Kim HH, Vijapurkar U, Hellyer NJ, Bravo D, Koland JG: Signal transduction by epidermal growth factor and heregulin via the kinase-deficient ErbB3 protein. Biochem J 1998, 334:189-195. 
8. Stove $C$, Bracke $M$ : Roles for neuregulins in human cancer. Clin Exp Metastasis 2004, 21:665-684.

9. Zhou BB, Peyton M, He B, Liu C, Girard L, Caudler E, Lo Y, Baribaud $\mathrm{F}$, Mikami I, Reguart N, et al:: Targeting ADAM-mediated ligand cleavage to inhibit HER3 and EGFR pathways in nonsmall cell lung cancer. Cancer Cell 2006, 10:39-50.

10. Warren CM, Kani K, Landgraf R: The N-terminal domains of neuregulin 1 confer signal attenuation. $J$ Biol Chem 2006, 281: 27306-27316.

11. Kani K, Warren CM, Kaddis CS, Loo JA, Landgraf R: Oligomers of ERBB3 have two distinct interfaces that differ in their sensitivity to disruption by heregulin. J Biol Chem 2005, 280: 8238-8247.

12. Burgess AW, Cho HS, Eigenbrot $C$, Ferguson KM, Garrett TP Leahy DJ, Lemmon MA, Sliwkowski MX, Ward CW, Yokoyama S: An open-and-shut case? Recent insights into the activation of EGF/ErbB receptors. Mol Cell 2003, 12:541-552.

13. Leahy DJ: Structure and function of the epidermal growth factor (EGF/ErbB) family of receptors. Adv Protein Chem 2004, 68:1-27.

14. Kohda D, Odaka M, Lax I, Kawasaki H, Suzuki K, Ullrich A, Schlessinger J, Inagaki F: A 40-kDa epidermal growth factor/transforming growth factor alpha- binding domain produced by limited proteolysis of the extracellular domain of the epidermal growth factor receptor. J Biol Chem 1993, 268:19761981.

15. Singer EM, Landgraf R, Horan T, Slamon DJ, Eisenberg D: Identification of a heregulin binding site in HER3 extracellular domain. J Biol Chem 2001, 276:44266-44274.

16. Kani K, Park E, Landgraf R: The extracellular domains of ErbB3 retain high ligand binding affinity at endosome $\mathrm{pH}$ and in the locked conformation. Biochemistry 2005, 44:15842-15857.

17. Kim JH, Saito K, Yokoyama S: Chimeric receptor analyses of the interactions of the ectodomains of ErbB-1 with epidermal growth factor and of those of ErbB-4 with neuregulin. Eur $J$ Biochem 2002, 269:2323-2329.

18. Ogiso $H$, Ishitani $R$, Nureki O, Fukai $S$, Yamanaka M, Kim JH, Saito K, Sakamoto A, Inoue M, Shirouzu M, Yokoyama S: Crystal structure of the complex of human epidermal growth factor and receptor extracellular domains. Cell 2002, 110:775-787.

19. Garrett TP, McKern NM, Lou M, Elleman TC, Adams TE, Lovrecz GO, Zhu HJ, Walker F, Frenkel MJ, Hoyne PA, et al.: Crystal structure of a truncated epidermal growth factor receptor extracellular domain bound to transforming growth factor alpha. Cell 2002, 110:763-773.

20. Elleman TC, Domagala T, McKern NM, Nerrie M, Lonnqvist B, Adams TE, Lewis J, Lovrecz GO, Hoyne PA, Richards KM, et al:: Identification of a determinant of epidermal growth factor receptor ligand-binding specificity using a truncated, highaffinity form of the ectodomain. Biochemistry 2001, 40:89308939.

21. Dawson JP, Berger MB, Lin CC, Schlessinger J, Lemmon MA, Ferguson KM: Epidermal growth factor receptor dimerization and activation require ligand-induced conformational changes in the dimer interface. Mol Cell Biol 2005, 25:7734-7742.

22. Garrett TP, McKern NM, Lou M, Frenkel MJ, Bentley JD, Lovrecz GO, Elleman TC, Cosgrove LJ, Ward CW: Crystal structure of the first three domains of the type-1 insulin-like growth factor receptor. Nature 1998, 394:395-399.

23. Cho HS, Mason K, Ramyar KX, Stanley AM, Gabelli SB, Denney DW, Leahy DJ: Structure of the extracellular region of HER2 alone and in complex with the Herceptin Fab. Nature 2003, 412:756-760.

24. Cho HS, Leahy DJ: Structure of the extracellular region of HER3 reveals an interdomain tether. Science 2002, 297:13301333 .

25. Ferguson KM, Berger MB, Mendrola JM, Cho HS, Leahy DJ, Lemmon MA: EGF activates its receptor by removing interactions that autoinhibit ectodomain dimerization. Mol Cell 2003, 11:507-517.

26. Li S, Schmitz KR, Jeffrey PD, Wiltzius JJ, Kussie P, Ferguson KM: Structural basis for inhibition of the epidermal growth factor receptor by cetuximab. Cancer Cel/ 2005, 7:301-311.

27. Bouyain S, Longo PA, Li S, Ferguson KM, Leahy DJ: The extracellular region of ErbB4 adopts a tethered conformation in the absence of ligand. Proc Natl Acad Sci USA 2005, 102:1502415029.
28. Garrett TP, McKern NM, Lou M, Elleman TC, Adams TE, Lovrecz GO, Kofler M, Jorissen RN, Nice EC, Burgess AW, et al.: The crystal structure of a truncated ErbB2 ectodomain reveals an active conformation, poised to interact with other ErbB receptors. Mol Cell 2003, 11:495-505.

29. Walker F, Orchard SG, Jorissen RN, Hall NE, Zhang H-H, Hoyne PA, Adams TE, Johns TG, Ward C, Garrett TPJ, et al:: CR1/CR2 interactions modulate the functions of the cell surface epidermal growth factor receptor. J Biol Chem 2004, 279:2238722398

30. Ozcan F, Klein P, Lemmon MA, Lax I, Schlessinger J: On the nature of low- and high-affinity EGF receptors on living cells. Proc Natl Acad Sci USA 2006, 103:5735-5740.

31. Wang Y, Pennock S, Chen X, Wang Z: Endosomal signaling of epidermal growth factor receptor stimulates signal transduction pathways leading to cell survival. Mol Cell Biol 2002, 22: 7279-7290.

32. Teis D, Wunderlich W, Huber LA: Localization of the MP1-MAPK scaffold complex to endosomes is mediated by p14 and required for signal transduction. Dev Cell 2002, 3:803-814.

33. Jiang G, Hunter T: Receptor signaling: when dimerization is not enough. Curr Biol 1999, 9:R568-R571.

34. Sorokin A, Lemmon MA, Ullrich A, Schlessinger J: Stabilization of an active dimeric form of the epidermal growth factor receptor by introduction of an inter-receptor disulfide bond. J Biol Chem 1994, 269:9752-9759.

35. Cao H, Bangalore L, Dompe C, Bormann BJ, Stern DF: An extra cysteine proximal to the transmembrane domain induces differential cross-linking of p185neu and p185neu. J Biol Chem 1992, 267:20489-20492.

36. Moriki T, Maruyama H, Maruyama IN: Activation of preformed EGF receptor dimers by ligand-induced rotation of the transmembrane domain. J Mol Biol 2001, 311:1011-1026.

37. Burke CL, Lemmon MA, Coren BA, Engelman DM, Stern DF: Dimerization of the p185neu transmembrane domain is necessary but not sufficient for transformation. Oncogene 1997, 14:687-696.

38. Zhang X, Gureasko J, Shen K, Cole PA, Kuriyan J: An allosteric mechanism for activation of the kinase domain of epidermal growth factor receptor. Cell 2006, 125:1137-1149.

39. Huang GC, Ouyang X, Epstein RJ: Proxy activation of protein ErbB2 by heterologous ligands implies a heterotetrameric mode of receptor tyrosine kinase interaction. Biochem J 1998, 331:113-119.

40. Jeffrey PD, Russo AA, Polyak K, Gibbs E, Hurwitz J, Massague J, Pavletich NP: Mechanism of CDK activation revealed by the structure of a cyclinA-CDK2 complex. Nature 1995, 376:313-320.

41. Berger MB, Mendrola JM, Lemmon MA: ErbB3/HER3 does not homodimerize upon neuregulin binding at the cell surface. FEBS Lett 2004, 569:332-336.

42. Mendrola JM, Berger MB, King MC, Lemmon MA: The single transmembrane domains of ErbB receptors self-associate in cell membranes. J Biol Chem 2002, 277:4704-4712.

43. Fleishman SJ, Schlessinger J, Ben-Tal N: A putative molecularactivation switch in the transmembrane domain of erbB2. Proc Natl Acad Sci USA 2002, 99:15937-15940.

44. Schaefer G, Akita RW, Sliwkowski MX: A discrete three-amino acid segment (LVI) at the C-terminal end of kinase-impaired ErbB3 is required for transactivation of ErbB2. J Biol Chem 1999, 274:859-866.

45. Landau M, Fleishman SJ, Ben-Tal N: A putative mechanism for downregulation of the catalytic activity of the EGF receptor via direct contact between its kinase and C-terminal domains. Structure 2004, 12:2265-2275.

46. Citri A, Gan J, Mosesson Y, Vereb G, Szollosi J, Yarden Y: Hsp90 restrains ErbB-2/HER2 signalling by limiting heterodimer formation. EMBO Rep 2004, 5:1165-1170.

47. Citri A, Harari D, Shohat G, Ramakrishnan P, Gan J, Lavi S, Eisenstein $M$, Kimchi A, Wallach D, Pietrokovski S, et al:: Hsp90 recognizes a common surface on client kinases. J Biol Chem 2006, 281:14361-14369.

48. Li H, Ruano MJ, Villalobo A: Endogenous calmodulin interacts with the epidermal growth factor receptor in living cells. FEBS Lett 2004, 559:175-180.

49. Li H, Sanchez-Torres J, Del Carpio A, Salas V, Villalobo A: The ErbB2/Neu/HER2 receptor is a new calmodulin-binding protein. Biochem J 2004, 381:257-266. 
50. McLaughlin S, Smith SO, Hayman MJ, Murray D: An electrostatic engine model for autoinhibition and activation of the epidermal growth factor receptor (EGFR/ErbB) family. J Gen Physiol 2005, 126:41-53.

51. Franklin MC, Carey KD, Vajdos FF, Leahy DJ, de Vos AM, Sliwkowski MX: Insights into ErbB signaling from the structure of the ErbB2-pertuzumab complex. Cancer Cell 2004, 5:317328.

52. Molina MA, Codony-Servat J, Albanell J, Rojo F, Arribas J, Baselga $\mathrm{J}$ : Trastuzumab (herceptin), a humanized anti-Her2 receptor monoclonal antibody, inhibits basal and activated Her2 ectodomain cleavage in breast cancer cells. Cancer Res 2001, 61: 4744-4749. 